\title{
In 7 Schritten zum Gerinnungs-Selbstmanagement
}

— Bei einer Therapie mit Vitamin-K-Antagonisten muss der INR-/\%Quickwert regelmäßig in der Arztpraxis bestimmt werden. Dies kann auch der Patient selbstständig zuhause im GerinnungsSelbstmanagement (GSM), z. B. mit dem CoaguChek ${ }^{\circledR}$-INRange-System, durchführen: Er kontrolliert seinen Gerinnungswert und passt bei Bedarf die Medikamentendosis entsprechend an. Lebensqualität und Therapieerfolg steigen.

Sind sich Arzt und Patient einig, dass das GSM infrage kommt, formuliert der Arzt eine Verordnungsbegründung zur Einreichung bei der Krankenkasse. Eine personalisierbare elektronische Vorlage hierfür steht auf www.coaguchek.de zur Verfügung. Messgerät und Teststreifen werden in der Regel von den gesetzlichen und privaten Krankenkassen übernommen, ebenso die Schulung zum GSM in anerkannten Schulungseinrichtungen.
Der Weg zum GSM führt immer über den behandelnden Arzt: Er berät den Patienten, meldet ihn bei einer Schulungseinrichtung an und stellt den Antrag bei der Krankenkasse. In 7 Schritten wird der Patient - mit Unterstützung seines Arztes - zum Gerinnungs-Selbstmanager:

- Am Anfang steht das Arzt-Patienten-Gespräch.

- Der Arzt befürwortet die Therapie und erstellt eine detaillierte Verordnungsbegründung.

- Der Arzt meldet den Patienten zur Schulung in einer nahegelegenen Schulungseinrichtung an. Dort erfolgt die Einweisung in das Messsystem, eine Unterweisung in die Anpassung der Medikamentendosis und das Training der Messung durch Blutentnahme aus der Fingerkuppe.
- Vor der Schulung klärt der Patient die Kostenübernahme mit seiner Krankenkasse ab.

- Der Patient besucht die Schulung und erhält ein Schulungszertifikat.

- Der Patient reicht das Schulungszertifikat gemeinsam mit der Verordnung des Arztes bei der Krankenkasse zur Kostenübernahme ein.

- Der Patient holt das CoaguChekINRange-System in der Apotheke oder im medizinischen Fachhandel ab.

Weitere Informationen zum GSM, ergänzende Patienteninformationen und Tipps zur Verordnung sowie individualisierbare Formulare stellt Roche Diagnostics unter der Telefonnummer 0800/0800855 oder auf der Website www.coaguchek.de bereit.

Red.

\section{Muskelrelaxanzien bei Rückenschmerzen}

\section{Verspannungen rationell behandeln}

— „Wir haben nach wie vor ein großes Problem mit muskuloskelettalen Erkrankungen“, bedauerte PD Dr. Michael Überall, Präsident der Deutschen Schmerzliga. Der Nürnberger Schmerztherapeut kritisierte, dass viele Therapien gewissermaßen „im Blindflug“ erfolgen, ohne rationale Grundlage.

\section{Die Patienten schnellstmöglich mobilisieren}

Überall plädierte für eine Mechanismenorientierte, auf die individuellen Patientenbedürfnisse zugeschnittene Behandlung. Ein pathologisch erhöhter Muskeltonus sei in einfachen klinischen Untersuchungen nachzuweisen und sollte entsprechend therapiert werden.

Gut verträgliche Muskelrelaxantien wie Methocarbamol (Ortoton') entspan- nen die verhärtete Skelettmuskulatur und ermöglichen dadurch eine raschere Mobilisierung. „Bringen Sie die Patienten so schnell wie möglich in die Bewegung“, appellierte Überall. Alle Medikamente seien nur Vehikel auf dem Weg dorthin.

Obwohl Methocarbamol seit über 40 Jahren im Einsatz ist, gibt es erst seit

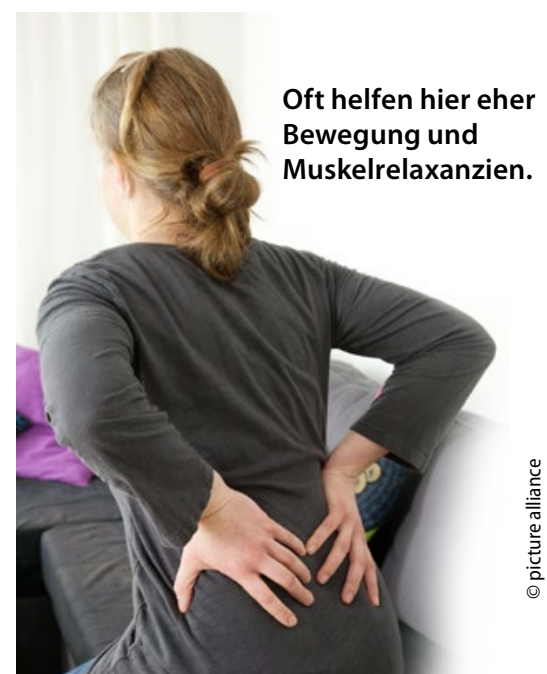

kurzem wissenschaftliche Evidenz für dessen gute Wirkung bei akuten und chronischen Rückenschmerzen. So ergab eine aktuelle retrospektive Analyse, dass neun von zehn Patienten mit mehrmonatiger Schmerzanamnese nach vierwöchiger Einnahme des Myotonolytikums $(3 \times 2$ Tabletten/d) einen Schmerzrückgang um 57-80\% erreichten.

„Das sind starke Antworten“, betonte Überall. Wenngleich sich die stärkste Response in der ersten Woche zeige, lohne es sich durchaus weiterzubehandeln (maximal 30 Tage), falls sich der gewünschte Therapieerfolg noch nicht eingestellt hat.

Dr. Martina-Jasmin Utzt

- Symposium „Muskelrelaxanzien bei Rückenschmerzen: mehr denn je aktuell", Innovationsforum Schmerzmedizin; Berlin, November 2016 (Veranstalter: Recordati) 